\title{
Stability of cellular patterns in directional solidification
}

\author{
John D. Weeks* and Wim van Saarloos ${ }^{\dagger}$ \\ AT\& T Bell Laboratories, Murray Hill, New Jersey 07974
}

(Received 19 July 1990)

\begin{abstract}
Ideas from the theory of constitutional supercooling, as well as an approximate linear stability analysis for small wavelength perturbations, lead to a simple stability criterion for the periodic cellular patterns seen in the directional solidification of alloys with small partition coefficient. There is good qualitative agreement with the limited experimental data available in the cellular regime. This criterion suggests that solutions found by using the analogy between directional solidification and Saffman-Taylor fingers may be unstable.
\end{abstract}

Recent analytical and numerical work has shown that the equations describing the directional solidification ${ }^{1}$ (DS) of a binary mixture admit a continuous family of periodic steady-state cellular solutions with varying wavelengths $\lambda .^{2-4}$ However, a steady-state analysis alone can give no information about the stability of these solutions, and hence their relevance for real experiments. In this paper we present an approximate stability analysis that in many cases will allow one to determine whether a given steady-state solution is likely to be stable. The simplest prediction is based on ideas from the theory of constitutional supercooling, ${ }^{1,5}$ and we refer to it as the local constitutional supercooling (LCS) criterion. The LCS criterion relates the positions of the tips of stable periodic cellular arrays in the experimental cell to the pulling velocity $V$, and its predictions can easily be checked experimentally. A more general, but still approximate, linear stability analysis for arrays of cellular patterns suggests that in many cases corrections to the LCS criterion should be small.

Equations similar to the LCS criterion have in fact been used before, particularly in the dendritic regime found at still higher pulling velocity, or smaller temperature gradient. ${ }^{6-8}$ However, the earlier derivations relied on somewhat ad hoc matching arguments, and they attempted to predict a unique pattern spacing for a given set of experimental conditions, rather than determine which members of a family of solutions might be stable. ${ }^{9}$ To our knowledge, the derivation and interpretation of the LCS criterion and its corrections in terms of stability concepts, and its particular relevance in the cellular regime has not been realized before.

We consider the standard one-sided solutal model, ${ }^{1}$ where solidification is assumed to occur as matter in a thin cell is pulled through a fixed linear temperature gradient, and impurity diffusion in the solid is ignored. The steadystate equations for DS in two dimensions in a frame moving at velocity $V$ can be written as ${ }^{1,4}$

$$
\begin{aligned}
& \nabla^{2} u+\frac{1}{l_{D}} \frac{\partial u}{\partial z}=0, \\
& \Delta u_{i} \cos \theta=-l_{D}(\hat{n} \cdot \nabla u)_{i}, \\
& u_{i}=1-z_{i} / l_{T}-d_{0} \kappa,
\end{aligned}
$$

where, using Eq. (3),

$$
\Delta u_{i} \equiv u_{i}-u_{i}^{s}=1-(1-k)\left(z_{i} / l_{T}+d_{0} \kappa\right) .
$$

Here $d_{0}$ is the chemical capillary length, $l_{T}$ the thermal length, $l_{D} \equiv D / V$ a diffusion length, $\kappa$ the interface curvature, and $\theta$ the angle between the interface normal $\hat{n}$ and the growth direction, taken parallel to the $z$ axis. ${ }^{10}$ A subscript $i$ denotes a quantity evaluated at the interface, and the superscript $s$ denotes the solid phase. The dimensionless field $u \equiv\left(c-c_{\infty}\right) / \Delta c_{0}$ measures the impurity concentration $c$ in the liquid relative to that far ahead of the interface $c_{\infty}$. It is normalized by the width of the planar two-phase region, $\Delta c_{0} \equiv c_{0}-c_{0}^{s}=c_{0}(1-k)$, with $k$ the partition coefficient and $c_{0}$ the concentration at the planar steady-state interface. Global conservation of impurities then fixes the location of the steady-state planar interface in the cell such that $c_{\infty}=k c_{0}$, or equivalently, $u_{0}=\Delta u_{0}=1$. This defines the origin $z=0$ of our coordinate system.

Equation (2) expresses local conservation of impurities as the interface advances, with $\Delta u_{i}$ in (4) the "impurity surplus" rejected at the interface. For materials with small partition coefficient $k$, we see that $\Delta u_{i}$ has a significant dependence on the position $z_{i}$ in the cell. This will play an important role in what follows.

Equation (3) takes account of surface-tension effects and imposes local equilibrium at the interface. For planar interfaces with $\kappa=0$, it describes the liquidus line

$$
u_{L}(z)=1-z / l_{T}
$$

which denotes the boundary of the stable single-phase region in a modified phase diagram where the abscissa is the reduced concentration field $u$ and the ordinate is the position $z$ in the cell (by assumption linearly related to the temperature $T$ ).

We note that in almost all cases, the curvature at the tips of experimentally relevant cellular patterns is small enough that the term $d_{0} \kappa$ in (3) represents only a very small correction. ${ }^{1,11}$ (This will be discussed more quantitatively later.) This suggests that we can think of the region near the tips as locally planar, and use some of the same stability ideas there that are used to examine the stability of the initial planar interface. The simplest approximation leads directly to the LCS criterion.

(C) 1990 The American Physical Society 
To begin the analysis, let us review the theory of constitutional supercooling (CS) for the planar interface. ${ }^{1,5}$ The basic idea is that in the steady state we can apply the concept of local thermodynamic equilibrium to the melt just in front of the moving planar interface. The interface is supposed to remain stable as long as the impurity concentration in the melt just in front lies in the stable single-phase region as determined from the phase diagram. Since we assume local equilibrium at the interface from (3), the CS stability criterion reduces to the following requirement. The (dynamical) gradient $|d u / d z|_{i}$ $=\Delta u_{i} / l_{D}$ determined from the boundary condition (2) must not exceed the liquidus slope $\left|d u_{L} / d z\right|=1 / l_{T}$ in (5). This criterion can be reexpressed as

$$
v \Delta u_{i} \leq 1
$$

where we define the standard dimensionless control parameter ${ }^{1} v \equiv l_{T} / l_{D}$. Note that $v$ is directly proportional to the velocity $V$.

The usual CS prediction $v_{c}^{\mathrm{CS}}=1$ for the stability limit of the plane ${ }^{1}$ follows from (6) where we note that global conservation requirements force the plane to be located at $z_{i}=0$, where $\Delta u_{i}=1$. This criterion is surprisingly accurate, given the simplicity of the CS argument. For materials with small $k$, a linear stability analysis (see below) predicts only small changes ${ }^{1}$ in the critical value of $v$, i.e., $v_{c} \approx 1$.

However, the arguments leading to (6) seem nearly as plausible as before when applied locally to the melt in front of the tips of certain (nonplanar) patterns, provided that the curvature corrections in Eq. (3) are small, and that $k$ is reasonably small, e.g., $k \lesssim 0.2$. In particular, we need to use only the boundary conditions (2) and (3) and the local symmetry in the tip region (nearly planar, with $\left.\theta_{t}=0\right)$ to arrive at (6), with $\Delta u_{i}=\Delta u_{t}$, the impurity surplus at the tip. When curvature corrections to $\Delta u_{t}$ from (4) are small, Eq. (6) can be reexpressed as

$$
\zeta_{t} \equiv(v-1)-(1-k) z_{t} / l_{D} \leq 0 .
$$

Equation (7) predicts that the tip position $z_{t}$ of stable patterns with $v>1$ must move $u p$ in the cell, relative to the planar position at $z=0$, until the smaller impurity surplus $\Delta u_{t}$ allows (6) or (7) to hold true. It is natural to conjecture that the operating point in real experiments should be close to the one that requires the least forward motion, i.e., the region where $\zeta_{t} \approx 0$. We refer to this as the LCS criterion. ${ }^{12}$

Of course, this argument is only suggestive. The CS condition (6) is not exact, even for the plane at $z=0$. It is easy to see that corrections to the LCS picture must surely be taken into account at large $v>1 / k$ (usually in the dendritic regime), since (7) would then predict an unphysical "concentration deficit" at the tip with $u_{t}<0$.

To determine the nature of the corrections to LCS, we extend the usual planar stability analysis ${ }^{1}$ to the case where the wavelength of interface perturbations is small compared to $\lambda$, the wavelength of the (steady-state) profile $z_{i}(x)$. Representing the perturbed interface by

$$
z_{i}^{q}(x, t)=z_{i}(x)+\hat{z}_{q} \exp (i q x+\omega t),
$$

we assume the perturbed impurity field takes the form

$$
u^{q}(x, z, t)=u(x, z)+\hat{u}_{q} \exp \left[i q x-p_{q}\left(z-z_{t}\right)+\omega t\right],
$$

where $u(x, z)$ is the field associated with the solution $z_{i}(x)$. In these and the equations that follow, we take the half wavelength $\lambda / 2$ as the unit of length and $\lambda^{2} / 4 D$ as the unit of time. We make the usual quasistationary approximation, ${ }^{1}$ where we assume that Eqs. (1) and (3) still hold, but use the correct (time-dependent) normal component of the interface velocity in Eq. (2). Substituting $z_{i}^{q}$ and $u^{q}$ and linearizing, we find in the region near $z_{t}$ our basic result

$$
\begin{aligned}
\omega= & -p^{2} k+\frac{\left(\partial^{2} u / \partial x^{2}\right)_{t}}{\Delta u_{t}} \\
& +\frac{p^{2}\left(p_{q} / p-1+k\right)\left[v \Delta u_{t}-1-(v-1) \sigma q^{2}\right]}{v \Delta u_{t}} .
\end{aligned}
$$

Here $p \equiv \lambda / 2 l_{D}$ is the Péclet number, which relates the scale of the pattern to the diffusion length, $p_{q}$ satisfies $p_{q}\left(p_{q}-p\right)=q^{2}$, and

$$
\sigma \equiv\left(\frac{\lambda_{s}^{0}}{\pi \lambda}\right)^{2}=\frac{4 v d_{0} l_{D}}{\lambda^{2}(v-1)},
$$

where $\lambda_{s}^{0}$ is the (lower) neutral stability wavelength for the plane in the limit as $k \rightarrow 0$. $\sigma$ provides a measure of the importance of surface-tension corrections due to the curvature of the interface. Experimental values for $\sigma$ are usually very small. . $^{11,13}$ We note that $(8)$ reduces to the standard result for the plane, ${ }^{1}$ where $\Delta u_{t}=\Delta u_{0}=1$, and $\left(\partial^{2} u / \partial x^{2}\right)_{t}=0$. The expression in (9) for $\lambda_{s}^{0}$ then follows from (8) if we set $k=0$ and solve for the associated wave vector $q_{s}^{0}$ giving $\omega=0$.

In general, for small $k$, the first term on the right-hand side of (8) is small, and important only in a small region of $v$ very near threshold. The quantity $\left(\partial^{2} u / \partial x^{2}\right)_{t}$ appearing in the second term on the right in (8) is negative (stabilizing) and numerical calculations indicate that it too is often small in magnitude. ${ }^{14}$

We can then obtain the LCS criterion from (8) by neglecting both these terms as well as all terms proportional to $\sigma{ }^{15}$ requiring that a stable solution with $\omega \leq 0$ results. This derivation makes it clear that the LCS condition $\zeta_{t} \approx 0$ gives an upper bound to the distance that the tips of patterns have to move to achieve stability, since all terms omitted in (8) are negative (stabilizing). Corrections due to the curvature of the interface are more important at large $v$, since they are proportional to $(v-1) \sigma$. Indeed, for very large $v$ in the dendritic regime, the tips move up very close to the line $z=l_{T}$. The stability is then determined by curvature effects (solvability) and weak interdendritic interactions. ${ }^{9}$ However, in the cellular regime with $v-1$ of $O(1)$, it appears that corrections to the LCS condition for patterns with small $\sigma$ are indeed small.

There is some limited experimental support for this conclusion. The only data we found in the literature that allowed us to test the LCS picture for cellular arrays are those of Esaka and $\mathrm{Kurz}^{7}$ on cells in succinonitrileacetone mixtures with $k=0.1$. In Fig. 1 we plot the predictions of Eq. (7) for the tip position as a function of the control parameter $v$, along with results obtained from the 


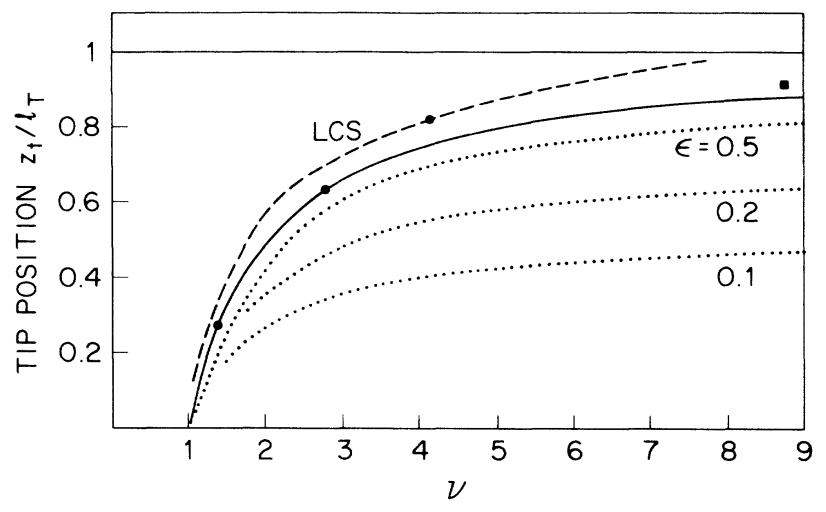

FIG. 1. Plot of the tip position $z_{t} / l_{T}$ vs $v$. The LCS criterion $\zeta_{t}=0$ for $k=0.1$ yields the dashed line. The solid line gives the $k \rightarrow 0$ limit of the LCS criterion. The dotted lines denote the predictions of (11) for $k=0.1$ and various values of $\epsilon$. The solid dot at $v=4.2$ and the square dot at $v=8.7$ represent two data points of Esaka and Kurz (Ref. 7), the latter showing some dendritic features, while the data points for $v=1.3$ and 4.2 are obtained from Ref. 8.

mapping onto the Saffman-Taylor finger as discussed below, and compare with the experimental data. There is good qualitative agreement between experiment and the LCS criterion for data in the cellular regime. Clearly, more experiments are called for, especially since wetting or surface preparation effects involving the cell thickness may be of importance. ${ }^{11}$

If the LCS picture is accurate, it has important consequences for the applicability to real experiments of the often used analogy between Saffman-Taylor fingers and DS at small Péclet number $p .^{2,16,17}$ To see this, let us first recall that the steady-state equations describing Saffman-Taylor (ST) fingering ${ }^{2}$ can be written as $\nabla^{2} \phi^{\mathrm{ST}}=0$, with boundary conditions $\left(\hat{n} \cdot \nabla \phi^{\mathrm{ST}}\right)_{i}=\cos \theta$ and $\phi_{i}^{\mathrm{ST}}$ $=\sigma^{\mathrm{ST}} \kappa$, where $\phi^{\mathrm{ST}}=-b^{2}\left(P-P_{0}\right) /(12 \mu V a)$, and $\sigma^{\mathrm{ST}}$ $=b^{2} \gamma / 12 \mu V a^{2}$. Here $b$ is the plate spacing, $\mu$ the viscosity, $V$ the finger velocity, $P$ the pressure field, $P_{0}$ the pressure inside the finger, $\gamma$ the surface tension, and $a$ the cell half width (taken as unit of length).

Dombre and Hakim ${ }^{2}$ show that for "fat" fingers whose width approaches the cell width $2 a, \sigma^{\mathrm{ST}}$ as defined here ${ }^{18}$ approaches the maximum value 1.393 , and they derive the limiting behavior for small $\epsilon$ of $\sigma^{\mathrm{ST}}(\epsilon)$, where $\epsilon$ is the relative groove width at the base of the finger. Karma and Pelcé ${ }^{16}$ give a convenient interpolation formula fitting numerical results for larger $\epsilon$. One finds that viscous fingers with narrow grooves are associated with values of $\sigma^{\text {sT }}$ of order unity.

Returning now to the DS problem, the analogy to viscous fingering can be seen most easily if we introduce a modified field ${ }^{2,17} \phi$ by the equation $u=1-p(z+\phi) / v$. The field $\phi$ measures the difference between the impurity concentration field $u$ and its dominant variation in the $z$ direction along a straight piece of the interface as dictated by the phase diagram. By substituting $\phi$ into Eqs. (1)-(3), when written in dimensionless units, we find exactly the modified DS equations $\nabla^{2} \phi+p \partial \phi / \partial z=-p$, with $\left[\zeta_{i}-(1-k) p(v-1) \sigma \kappa\right] \cos \theta=(\hat{n} \cdot \nabla \phi)_{i} \quad$ and $\quad \phi_{i}=(v$
$-1) \sigma \kappa$. Here $\zeta_{i} \equiv(v-1)-(1-k) p z_{i}$ is the LCS parameter (7) evaluated at a general (dimensionless) position $z_{i}$.

These equations are valid for any $p$, but we now take the limit $p \rightarrow 0$ in the following natural way. ${ }^{17} \mathrm{We}$ examine the region near the tips, allowing for the possibility that $p z_{t}$ reaches a finite limit as $p \rightarrow 0$, but make the important assumption, to be verified later, that $\zeta_{t}$ remains greater than zero. In the fingertip region, of $O(1)$ in our units, we can then ignore the $z$ variation of $\zeta_{i}$, replacing it by $\zeta_{t}$. Then ignoring all other terms proportional to $p$, we see the modified DS equations are identical in form to the ST equations given above if we let $\phi / \zeta_{t}=\phi^{\mathrm{ST}}$ and

$$
\frac{(v-1) \sigma(p \rightarrow 0)}{\zeta_{t}(p \rightarrow 0)}=\sigma^{\mathrm{ST}} \text {. }
$$

Karma and Pelcé ${ }^{16}$ and Mashaal et al. ${ }^{17}$ use conservation and matching arguments to arrive at an expression for $\zeta_{t}$. Using also (10), their result can be expressed as

$$
\frac{\sigma(p \rightarrow 0)}{\sigma^{\mathrm{ST}}}=\frac{\zeta_{t}(p \rightarrow 0)}{v-1} \approx \frac{1}{1+(1-k) \epsilon / k} .
$$

Different members of the family of solutions satisfying (10) and (11) are parameterized by the relative groove width $\epsilon$ of the DS finger, now measured in the "matching region," a distance of $O(1)$ behind $z_{t}$. Equation (11) predicts that for small $\epsilon \lesssim k$, both $\zeta_{t} /(v-1)$ and $\sigma$ are $O(1)$. This is consistent with a key assumption made in the mapping to the ST problem: $\zeta_{t}$ remains finite as $p \rightarrow 0$.

Weeks and van Saarloos ${ }^{4}$ showed that if the partition coefficient $k$ is small, there is a natural extension of the matching arguments leading to (10) and (11) to systems with finite $p$ of $O(1)$. The resulting finite $p$ corrections to the right-hand side of (11) are relatively small, at least for small $\epsilon$, and yield somewhat larger values for $\zeta_{t}$ and $\sigma$.

Unfortunately, this entire class of solutions appears to be in qualitative disagreement with many experiments. Equation (11) implies that the small $\epsilon$ solutions are associated with large values of $\sigma$. Note that solutions with $\sigma>\pi^{-2}$ have wavelengths $\lambda<\lambda_{s}^{0}$ lying outside the planar instability band. However, most experimental patterns have narrow grooves, or small $\epsilon$, but with small $\sigma$ values such that $\lambda>\lambda_{s}^{0}{ }^{11,13}$ Small values of $\sigma$ can arise from (11), but only for large $\epsilon$.

Moreover, these solutions are not consistent with the LCS picture, where we expect $\zeta_{t} \approx 0 .{ }^{19}$ This is illustrated in Fig. 1, where we note that the ST curves for narrow grooves, say $\epsilon \lesssim 0.1$, lie far below that of the LCS criterion. Of course, this argument is not conclusive, especially since corrections to the LCS criterion are more important at large $\sigma$ and all these tend to increase the value of $\zeta_{t}$. Still, it does suggest there could exist a different, and more physically relevant, branch of steady-state solutions where $\zeta_{t}$ is small for small $\epsilon$. Note from (11) that for small $p$ this would also imply small values of $\sigma$ as seen in experiments.

This conclusion seems fully in accord with the work of Brener, Geilikman, and Temkin ${ }^{20}$ on the related problem of crystal growth in a channel (essentially the $v \rightarrow \infty$ limit of DS). When interface kinetics is taken into account, 
they find at least two branches of steady-state solutions. Only one of these is related to ST fingers in the limit $p \rightarrow 0$, but it exhibits several unphysical features. The second branch properly described free dendritic behavior as the channel width tends to infinity. We believe ${ }^{4}$ a similar scenario may apply to DS.

These results point out the need for a systematic experimental test of the LCS criterion, and its corrections, for cellular arrays. If this turns out to be accurate, then there are theoretical as well as practical implications. Many theoretical steady-state patterns found in the literature are predicted to be unstable by the LCS criterion. The considerable body of work based on the mapping to the ST finger should then be reexamined, and a more rigorous and systematic stability analysis for arrays of cellular patterns is called for. More generally, the question of "branch selection" for this entire class of pattern forming systems needs more study.
*Present address: Institute for Physical Science and Technology, University of Maryland, College Park, MD 20742.

${ }^{\dagger}$ Address after 1 January 1991: Instituut Lorentz, University of Leiden, Nieuwsteeg 18, 2311 SB Leiden, The Netherlands.

${ }^{1}$ For a general review, see J. S. Langer, Rev. Mod. Phys. 52, 1 (1980).

${ }^{2}$ T. Dombre and V. Hakim, Phys. Rev. A 36, 2811 (1987). See also M. Ben-Amar and B. Moussallam, Phys. Rev. Lett. 60, 317 (1988).

${ }^{3}$ See, e.g., D. A. Kessler and H. Levine, Phys. Rev. A 39, 3041 3208 (1989); N. Ramprasad, M. J. Bennett, and R. A. Brown, Phys. Rev. B 38, 583 (1988).

${ }^{4}$ J. D. Weeks and W. van Saarloos, Phys. Rev. A 39, 2772 (1989). A more complete description of both the matching methods used in this reference, and the LCS ideas discussed herein can be found in J. D. Weeks, W. van Saarloos, and M. Grant (unpublished).

${ }^{5}$ W. A. Tiller, K. A. Jackson, J. W. Rutter, and B. Chalmers, Acta Metall. 1, 428 (1953).

${ }^{6}$ See, e.g., D. J. Allen and J. D. Hunt, Metall. Trans. 7A, 767 (1976), and references therein.

${ }^{7}$ H. Esaka and W. Kurz, J. Cryst. Growth 72, 578 (1985). Some additional data from Esaka is reported in Ref. 8.

${ }^{8}$ B. Billia, H. Jamgotchian, and R. Trivedi (unpublished).

${ }^{9}$ This point was made by J. A. Warren and J. S. Langer (unpublished), who studied the stability of dendritic arrays.

${ }^{10}$ Here $d_{0} \equiv \gamma T_{M} /\left(L m_{L} \Delta c_{0}\right)$ with $\gamma$ the surface tension, $L$ the latent heat per unit volume, $T_{M}$ the melting temperature of the pure solvent, and $m_{L}$ the liquidus slope $\left|d T_{L} / d c\right|$; and $l_{T} \equiv m_{L} \Delta c_{0} / G$, with $G$ the temperature gradient.

"S. de Cheveigné, C. Guthmann, and M. Lebrun, J. Phys. (Paris) 47, 2095 (1986)

${ }^{12}$ In general, let $\mathbf{r}_{i} \equiv\left[x_{i}(z), z\right]$ denote an arbitrary point on the interface of a fingerlike pattern, and $\mathbf{r}_{\alpha} \equiv \mathbf{r}_{i}+\alpha l_{T} \hat{n}$ a point in the adjacent fluid layer for a small normal displacement $\alpha l_{T}$ with $\alpha \ll 1$. A measure of the differential supercooling at $\mathbf{r}_{a}$ is $\delta u_{\alpha}^{\mathrm{LCS}} \equiv\left[u_{L}\left(\mathbf{r}_{\alpha}\right)-u\left(\mathbf{r}_{a}\right)\right] / \alpha \approx \zeta(z) \cos \theta$. Here $\zeta(z)$ is (7) evaluated at the general position $z$, and we have used (5) and (2), ignoring curvature corrections. It is easy to show from the Scheil equation (Ref. 4) that $\delta u_{\alpha}^{\mathrm{LCS}} \rightarrow 0$ deep in the grooves due to the $\cos \theta$ factor, but this fact places no $\operatorname{con}$ straints on the absolute position of the finger. However, requiring that the differential supercooling is small near the tip, where $\cos \theta=1$, yields the LCS criterion, which does (approximately) fix $z_{t}$.

${ }^{13}$ For example, using the data shown in Fig. 2 of Ref. 11, after some misprints are corrected, we find $\sigma \approx 0.006$.

${ }^{14}$ See, e.g., L. H. Ungar and R. A. Brown, Phys. Rev. B 29, 1367 (1984); 31, 5931 (1985). By differentiating (3), we can show that $\left(\partial^{2} u / \partial x^{2}\right)_{t}=-(p / v)\left[v \Delta u_{t}-1-(v-1) \sigma(d \kappa / d z)_{t}\right] \kappa_{t}$, noting that $d u_{i} / d z=(\partial u / \partial z)_{i}+(\partial u / \partial x)_{i} /\left(d z_{i} / d x\right)$ and that near $x_{t},(\partial u / \partial x)_{1}=\left(\partial^{2} u / \partial x^{2}\right)_{t}\left(x_{i}-x_{t}\right)$, etc. Thus the idea that $v \Delta u_{t}-1 \approx \zeta_{t}$ is small is consistent with small $\left(\partial^{2} u /\right.$ $\left.\partial x^{2}\right)$.

${ }^{15}$ Although short-wavelength perturbations were assumed in deriving (8), we expect it will remain at least qualitatively accurate for perturbation wavelengths of order $\lambda$, i.e., $q \approx \pi$. For such perturbations, the term $(v-1) \sigma q^{2}$ in $(8)$ represents only a small correction in the cellular regime if $\sigma$ is small. However, since (8) does not properly incorporate the physics of competition between different fingers generated by perturbations with still smaller wave vectors $q$, it cannot be used to give a quantitative treatment of the pattern selection mechanism.

${ }^{16}$ See A. Karma and P. Pelcé, Phys. Rev. A 39, 4162 (1989), and references therein.

${ }^{17}$ M. Mashaal, M. Ben-Amar, and V. Hakim, Phys. Rev. A 41, 4421 (1990).

${ }^{18} \mathrm{Our} \sigma^{\mathrm{ST}}$ is equal to $d_{0} / \pi^{2}$ in the notation of Ref. $2 ; 4 / C$ in Eq. (22) of Ref. 16; and $4 \sigma_{\mathrm{ST}}$ in Ref. 17.

${ }^{19}$ Indeed, if $\zeta_{t} \leq 0$, or even if it is $O(p)$ as $p \rightarrow 0$, then the mapping to the ST equations could not be carried out. However, it seems more likely to us that the problem with the matching result (11) [and our generalization (Ref. 4) to finite $p$ ] lies in a breakdown of the assumption that there is a single matching region whose width vanishes as $\epsilon \rightarrow 0$ where the inner (tail) and outer (tip) solutions can be joined together.

${ }^{20}$ E. A. Brener, M. Geilikman, and D. E. Temkin, Zh. Eksp. Teor. Fiz. 94, 241 (1988) [Sov. Phys. JETP 67, 565 (1988)]. 